\title{
SUR LA PRÉSENCE DE CRASPEDACUSTA SOWERBYI LANK., MÉDUSE D'EAU DOUCE, DANS LA RÉGION DE MONTEREAU
}

\author{
par M. GIRARD et F. POPLIN
}

\begin{abstract}
Depuis qu'a commencé en 1964 l'exploitation du gisement préhistorique de PINCEVENT (commune de la Grande Paroisse) sur la rive gauche de la Seine à sept kilomètres en aval de Montereau, nous avons, au sein de l'équipe que dirige Monsieur LEROI-GOURHAN, Professeur de Préhistoire au Collège de France, la possibilité d'observer chaque été, pendant les moments de loisir, la faune locale, et particulièrement celle du lac artificiel.
\end{abstract}

C'est ainsi que nous y avions remarqué, il y a quelques années, la présence de Dreissensia polymorpha, petite moule d'eau douce à byssus originaire d'Europe centrale. A l'époque, ce plan d'eau communiquait encore avec le fleuve, où sévissait sur les poissons la bucéphalose dans sa phase la plus forte. L'absence du Mollusque et celle de la maladie parasitaire dans I'Yonne en amont d'Auxerre d'une part, leur présence conjointe dans la région de Montereau d'autre part avait mené l'un de nous (F. P.) à l'hypothèse que le Mollusque pourrait intervenir comme hôte intermédiaire dans le cycle de développement du parasite, ce que rendait encore plus probable leur commune origine centreuropéenne. II s'en était ouvert à son confrère DE KINKELIN qui put tirer parti de cette observation dans l'étude qu'il faisait sur cette maladie, et qui démontra que cette apparence reflétait une réalité.

Cet été est survenu dans la même pièce d'eau un hôte assez inhabituel pour qu'il vaille la peine d'être signalé, comme nous y ont encouragés Monsieur FRANC, Professeur au Muséum d'Histoire Naturelle et Monsieur VIVIER, Directeur de la Station d'Hydrobiologie Continentale, que nous sommes heureux de remercier ici pour la chaleur de leur accueil.

C'est au cours d'une séance d'entrainement à la plongée sous-marine que l'un des signataires (M. G.) remarqua les méduses. Elles se tenaient près du fond, et ce sont les mouvements d'eau qui, en les soulevant, permirent de les découvrir. Assez abondantes, elles ne formaient cependant pas les amas nuageux qui ont été parfois observés ailleurs. Ce sont des individus femelles, de un à deux centimètres de diamètre. Non spécialistes, nous nous bornerons à signaler 
cette station, réservant à d'autres une étude plus approfondie. C'est dans le domaine du milieu que PINCEVENT semble devoir être le plus intéressant. Le lac communiquait naguère encore avec la Seine où s'en allaient les péniches chargées de sable; il a été en effet entièrement creusé par la drague, qui entretenait dans l'eau un limon opaque interdisant toute flore macroscopique. Ces travaux ont été interrompus, une digue a fait de la carrière une eau close, les bourbes sont tombées et l'on voit d'année en année la végétation prendre ses droits.

C'est donc dans une évolution écologique manifeste que s'inscrit l'apparition de Craspedacusta Sowerbyi à Pincevent.

Más s'agit-il bien d'une apparition? Si la méduse était rare les années précédentes, ou plus encore occulte et potentielle sous forme d'hydraires, il n'est pas certain qu'elle ne nous ait pas échappé, d'autant que notre attention n'était pas préparée A dire vrai, c'est plutôt son abondance qui est cause de la découverte, de sorte qu'il vaudrait mieux parler de révélation que d'apparition. Et cela vaut pour la majorité des observations antérieures : ce n'est qu'au

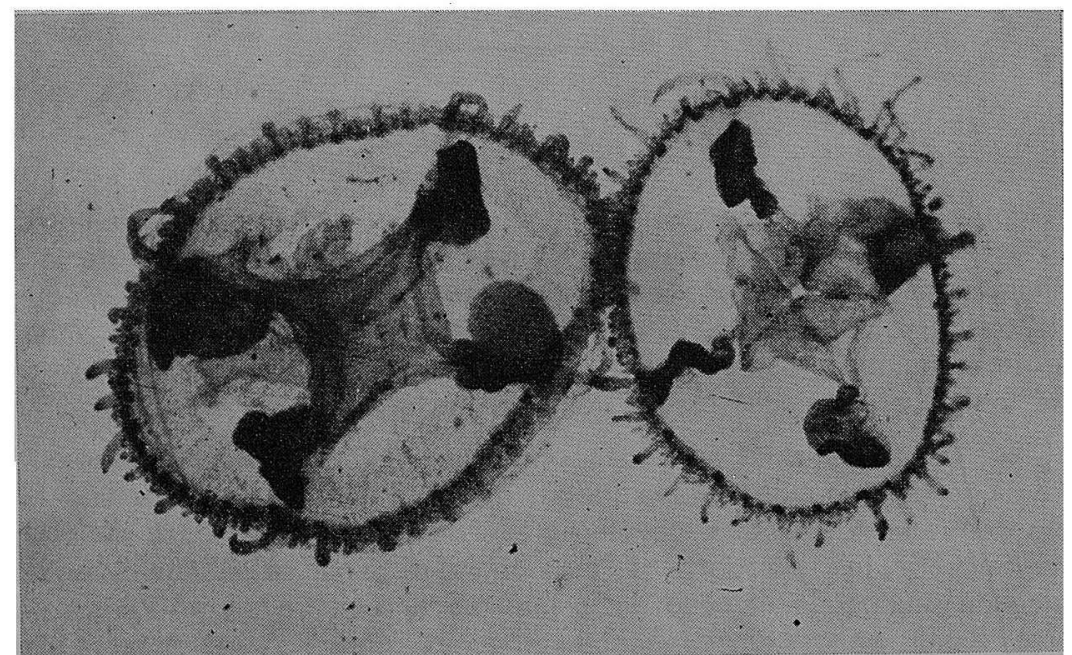

(photo R. Barbier)

Méduses d'eau douce pêchées à Pincevent

niveau de phénomène bien visible que l'animal entre dans le champ de perception, c'est-à-dire quand il est très abondant. Cela a pu accréditer la thèse de l'apparition subite et massive. En fait, comme le montre STADEL ( $\left.{ }^{*}\right)$, l'investigation systématique aboutit à trouver au long d'une rivière de nombreuses stations, lorsqu'il en a été signalée une. En France, les découvertes sont fortuites o: génóralement liées à la présence d'un naturaliste averti. La discrétion de cette espèce tient pour beaucoup à sa situation de membre égaré d'une famille marine : nombre de pêcheurs doivent la voir sans la remarquer, n'ayant pas de référence visuelle comme ils en auraient pour un nouveau poisson (comme le Sandre). Ajoutons qu'ils ne songent généralement pas à en faire

${ }^{*}$ ) STADEL (O). Neuere Kenntnisse über die Okologie und Verbreitung der Süsswassermedusa Craspedacusta Sowerbyi. ABH. NATURW. VER. Hamburg N.F. 5 (fév. 1960) 1961 : 157-192 4 cartes. 
mention comme ici. Aussi la raison principale de ces lignes est-elle d'avertir le lecteur et de le prier de bien vouloir signaler les observations de méduse d'eau douce qu'il pourrait faire à la Station d'Hydrobiologie Continentale, 14, avenue de Saint-Mandé, Paris 12e, ou au Bulletin qui transmettra.

Déjà, nous devons à M. JAMAIN, technicien de la Station d'hydrobiologie de l'E.D.F. à la Grande-Paroisse (S.-et-M.), de savoir que Craspedacusta Sowerbyi existait sous sa forme méduse

- en été 1962 dans la carrière de la Tombe, dans la vallée de la Seine à quinze kilomètres en amont de Montereau, pièce d'eau qui avait communiqué avec le fleuve, mais qui était close à l'époque;

- au même moment dans la carrière de Cannes-Ecluse, en communication avec I'Yonne quelques kilomètres en amont du confluent;

- en été 1969, également dans une ancienne balastière, close, près de la Centrale E.D.F., à quatre kilomètres en aval de Pincevent.

On aura noté au passage que des méduses ont été vues dans une pièce d'eau communiquant avec l'Yonne, et par elle avec la Seine, ce qui ouvre ia possibilité d'en trouver dans ces cours d'eau.

Il semble que ce soit la première fois qu'on signale la méduse dans ce bassin hydrographique : on avait seulement trouvé l'hydraire dans l'Oise en 1944.

Voici la récapitulation des découvertes en France, par ordre chronologique. Nous avons tenu en main tous les textes les relatant, mais il serait trop long d'en donner la liste, qu'on retrouvera dans STADEL et dans BOUILLON (Etude monographique du genre Limnocnida. Limnoméduse, Ann. Soc. Roy. Zool. Belgique, 87 (2) 1956-57).

\begin{tabular}{|c|c|c|}
\hline 01 & $\begin{array}{l}\text { Parc de la Tête d'Or à Lyon } \\
\text { dans le Dropt (33) vers Monségur }\end{array}$ & $\begin{array}{l}\text { (Vaney et Conte 1901) } \\
\text { (Feytaud et Cadenat 1930) }\end{array}$ \\
\hline 33 & dans la Mayenne à Château-Gontier & (Germain 1934) \\
\hline 33 & Dropt (même lieu) & (Feytaud 1934) \\
\hline 43 & Faculté Sciences Lyon (aquarium) & (de Larambergue 1945) \\
\hline 44 & dans l'Oise à Précy & (Fauré-Fremiet 1945) \\
\hline 45 & dans le Canal du Midi à Toulouse & (Despax 1945) \\
\hline 49 & Dropt (même lieu) & (Weill 1949) \\
\hline 49 & dans le Thouet (79) et la Vienne & (de Larambergue et Origny 1955) \\
\hline 50 & dans le Canal de Bourgogne (21) & (Denis 1950) \\
\hline & dans l'Auxance vers Poitiers (86) & (de Larambergue et Origny 1955) \\
\hline 52 & dans le Lot à Aiguillon (47) & \\
\hline 59 & dans le Rhône à Viviers (7) & (Serra-Tosio 1961) \\
\hline & dans un étang près de Grenoble & » \\
\hline 60 & même lieu & 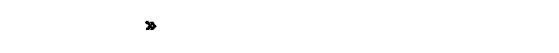 \\
\hline & dans l'Allan près de Montbéliard (25) & (Acolat 1961) \\
\hline & dans la Têt (66) & \\
\hline
\end{tabular}

